Tropical Journal of Pharmaceutical Research June 2012; 11 (3): 335-343

(c) Pharmacotherapy Group, Faculty of Pharmacy, University of Benin

Benin City, 300001 Nigeria.

All rights reserved.

Available online at http://www.tjpr.org

Research Article

http://dx.doi.org/10.4314/tjpr.v11i3.1

\title{
Formulation of Bioadhesive Carbomer Gel Incorporating Drug-Loaded Gelatin Microspheres for Periodontal Therapy
}

\author{
K Karthikeyan ${ }^{1}$, R Durgadevi ${ }^{1}$, K Saravanan ${ }^{1}$, K. Shivsankar ${ }^{1}$, S. Usha ${ }^{2}$ and \\ M Saravanan ${ }^{2^{*}}$ \\ ${ }^{1}$ Vels College of Pharmacy, Pallavaram, Chennai, India, ${ }^{2}$ Jeffrey Cheah School of Medicine and Health Sciences, Monash \\ University, Sunway Campus, Malaysia 46150.
}

\begin{abstract}
Purpose: To formulate carbomer gel to localize and target drug action in periodontal pockets.

Methods: Diclofenac sodium (DS, anti-inflammatory) and metronidazole hydrochloride ( $\mathrm{MH}$, antibacterial) were loaded in gelatin microspheres using glutaraldehyde cross-linking. The microspheres were evaluated for drug loading, entrapment and encapsulation efficiency, particle size, drug release as well as by scanning electron microscopy (SEM) and differential scanning calorimetry (DSC). The microspheres were incorporated into carbomer gel and evaluated for drug release.

Results: Loading, entrapment and encapsulation efficiencies of DS in the microsphere were 23.4, 93.6, and $74.9 \mathrm{w} / \mathrm{w} \%$, respectively, while for $\mathrm{MH}$ the values were $21.5,86.0$ and $73.1 \% \mathrm{w} / \mathrm{w}$, respectively. Mean particle size of unloaded microspheres, DS- and MH-loaded microspheres was 33.5, 67.8 and $51.4 \mu \mathrm{m}$, respectively. SEM showed spherical geometry of microspheres while DSC indicated the amorphous nature of $t$ entrapped $D S$ and $M H$. Sustained release of DS and MH over a 4-h period from the microspheres and gel was achieved.

Conclusion: Carbomer gel loaded with microspheres of diclofenac and metronidazole is a potential localized delivery system for the treatment inflammation and infection in periodontal pockets.
\end{abstract}

Keywords: Gelatin microspheres, Bioadhesive dosage forms, Metronidazole, Diclofenac, Periodontal disease, Sustained release 


\section{INTRODUCTION}

Periodontitis is an inflammatory disease caused by chronic bacterial infection of the gum and bone supporting the teeth $[1,2]$. Antimicrobial agents such as metronidazole, tetracycline and erythromycin are used to treat bacterial infections in periodontal diseases [3]. Non-steroidal anti-inflammatory drugs (NSAIDs) such as ibuprofen and diclofenac sodium are used to treat pain and inflammation. These drugs are administered locally as gel or given orally in the form of tablets. Due to their short half life, these medications have to be taken frequently to maintain the desired therapeutic effect.

In periodontal diseases, both drugs are used simultaneously to treat infection and inflammation. A single delivery system which can deliver both drugs is expected to be more convenient, beneficial and improve patient compliance. Hence in the present investigation, we have attempted to develop a gel formulation loaded with diclofenac sodium and metronidazole. Since the preparation has the same base at the time of administration, gel containing diclofenac sodium and metronidazole can be simply mixed and applied to the periodontal cavity with a special dispenser [4] used by the periodontist to deliver medication or gel formulation into periodontal pockets.

Though microspheres loaded with antimicrobials have been reported and are currently available in the market [5], none includes microspheres loaded with both an anti-inflammatory and antimicrobial drugs. Sustained release formulations will be beneficial to patients with gastric ulceration or sensitivity to the drug, as the drug effect would be localized. Administration of the conventional drug delivery systems of metronidazole leads to side effects such as nausea and vomiting [6].

Carbomer (carboxy vinyl polymer) is a very high molecular weight polymer of acrylic acid with cross linkages of allyl sucrose [7]. Due to the high proportion of the carboxy groups present, carbomer solution is known to be acidic. It is also of low viscosity but when neutralized with triethanolamine, it is converted to highly viscous gels. The adhesive prosperities of carbomer are exploited to develop bioadhesive gels and drug delivery systems for controlled and localized drug delivery [8-10]. .

To obtain a sustained and targeted delivery, drugs can encapsulated in gelatin microspheres, which are then formulated as a gel using carbomer. To overcome problems associated with the conventional system, we aimed to develop a bioadhesive gel formulation which can sustain and localize the drug in the periodontal pockets for an effective treatment.

\section{EXPERIMENTAL}

\section{Materials}

Gelatin (type B, 300 bloom strength) was purchased from Sigma Chemicals, USA. Carbopol 934 and triethanolamine GR (99 \% purity) were purchased from Loba Chemie, India. Metronidazole hydrochloride and diclofenac sodium were gifts from Arvind Remedies, Thiruvellore, Chennai, India. Glutaraldehyde and isopropyl alcohol were obtained from S.D. Fine Chemicals Ltd., Mumbai, India. Span 80, Tween 80, acetone and toluene were purchased from Indian Research Products, Chennai, India.

\section{Preparation of gelatin microspheres}

Three grams of gelatin were accurately weighed and mixed in $10 \mathrm{ml}$ of distilled water; preheated to $60{ }^{\circ} \mathrm{C}$, followed by the addition of Tween $80(0.1 \% \mathrm{w} / \mathrm{v})$. To this, $1 \mathrm{~g}$ of diclofenac sodium was added and thoroughly mixed to obtain a homogeneous solution. The mixture was maintained at $50{ }^{\circ} \mathrm{C}$, and then added drop wise into $100 \mathrm{ml}$ of sesame oil containing Span $80(0.1 \% \mathrm{w} / \mathrm{v})$ preheated to $60{ }^{\circ} \mathrm{C}$ while stirring at $1000 \mathrm{rpm}$ with a 3blade stirrer (Remi, India) in order to form a 
w/o emulsion. Toluene $(0.5 \mathrm{ml})$ saturated with glutaraldehyde was added drop-wise to the emulsion and stirring and stirred for $1 \mathrm{~h}$ at room temperature to stabilize the microspheres. The mixture was then left to cool at between $5-10{ }^{\circ} \mathrm{C}$ for $30 \mathrm{~min}$ to enhance settling of the microspheres. The microspheres were collected by filtration using Whatman filter paper (no. 41) and washed with $3 \times 10 \mathrm{ml}$ of chloroform followed by $2 \times 10 \mathrm{ml}$ of $5 \% \mathrm{w} / \mathrm{v}$ sodium metabisulphite. Finally, the microspheres were washed with $10 \mathrm{ml}$ of acetone, dried at room temperature and transferred to glass vials that were stored in desiccators until used. Similarly, microspheres loaded with metronidazole and unloaded microspheres were formulated and stored in desiccators.

\section{Preparation of gel}

Preparation of the carbomer gel was based on a previously reported composition and method [7]. Accurately weighed diclofenac sodium $(100 \mathrm{mg}$ ) was added to $15 \mathrm{ml}$ of water in a beaker and stirred well to dissolve the drug; $400 \mathrm{mg}$ of carbopol was dissolved in this drug solution. Gelatin microspheres loaded with $100 \mathrm{mg}$ of diclofenac sodium was added to the drug-carbopol solution and mixed well. Methyl hydroxybenzoate $(0.15$ $\% w / w)$, propyl hydroxybenzoate $(0.05 \% \mathrm{w} / \mathrm{w})$ and sodium metabisulphate $(0.1 \% \mathrm{w} / \mathrm{w}))$ were then added to microsphere/drug/carbopol mixture, while stirring at $250 \mathrm{rpm}$, to obtain a homogenous mixture. Stirring was continued until a lump-free suspension was obtained. Thereafter, $0.33 \mathrm{ml}$ of triethanolamine was added to produce a gel. This was followed by the addition of a sweetening agent (saccharin sodium, $0.1 \% \mathrm{w} / \mathrm{w}$ ) and more water to make up to $20 \mathrm{~g}$ of gel. Similarly, a gel containing metronidazole hydrochloride was prepared. The amount of metronidazole used was the same as that of diclofenac.

\section{Determination of drug content}

The amount of diclofenac sodium presents in the gelatin microspheres was determined by digestion with $1 \mathrm{M}$ sodium hydroxide [11].
Briefly, $100 \mathrm{mg}$ of microspheres was dispersed in $100 \mathrm{ml}$ of $1 \mathrm{M}$ sodium hydroxide in a $100 \mathrm{ml}$ standard flask. and kept overnight for $12 \mathrm{~h}$. It was then filtered, diluted and diclofenac sodium content was determined spectrophotometrically (Shimadzu 1601) at $276 \mathrm{~nm}$. Sodium hydroxide (M) was used as blank. The amount of metronidazole present in gelatin microspheres was determined by digestion with hydrochloric acid [12]. Briefly, $100 \mathrm{mg}$ of microspheres was dispersed in $100 \mathrm{ml}$ of $1 \mathrm{M}$ hydrochloric acid in a $100 \mathrm{ml}$ standard flask and kept overnight for $12 \mathrm{~h}$. It was then filtered, diluted and metronidazole hydrochloride content was determined at 320 $\mathrm{nm}$. Hydrochloric acid (1M) was used as blank.

\section{Determination of percentage of drug loading}

The percentage of diclofenac sodium and metronidazole hydrochloride loading in microspheres can be estimated using Eq 1 .

$$
L=\frac{Q_{m}}{W_{m}} \times 100
$$

where, $L$ is the loading (\%) of microspheres, $Q_{m}$ is the quantity of diclofenac sodium and metronidazole hydrochloride present in $\mathrm{W}_{\mathrm{m}}$ of microspheres and $W_{m}$ is the weight of the microspheres in grams

\section{Determination of encapsulation efficiency}

The amount of diclofenac sodium and metronidazole hydrochloride encapsulated in the microspheres was determined using $\mathrm{Eq}$ 2.

$$
E=\frac{Q_{p}}{Q_{t}} \times 100
$$

where $\mathrm{E}$ is encapsulation efficiency (\%), $Q_{p}$ is the quantity of drug encapsulated in the microspheres $(\mathrm{g}), Q_{t}$ is the actual quantity of drug used for encapsulation ( $\mathrm{g}$ ), and $Q_{p}$ is the product of drug content per gm of microspheres and yield of microspheres $(\mathrm{g})$. 


\section{Particle size analysis}

The microspheres were analyzed for size and size distribution by first dispersing them in 20 $\% \mathrm{v} / \mathrm{v}$ isopropyl alcohol to avoid swelling, vortexing for $3 \mathrm{~min}$ and ultrasonicating for 30 $\mathrm{s}$ before sampling. The particle size was measured by laser diffraction (Shimadzu Sald 1100, Japan) and plotted for size distribution using the software supplied by the manufacturer.

\section{Scanning electron microscopy}

The sample the scanning electron microscopy (SEM) analysis was prepared by sprinkling the microspheres on one side of the double adhesive stub. The stub was then coated with gold using Jeol JFC 1100 sputter coater. The SEM analysis of the microspheres was carried out using Jeol JSM 5300 , Japan. The microspheres were viewed at an accelerating voltage of $15-20 \mathrm{kV}$.

\section{Differential scanning calorimetry}

Differential scanning calorimetry (DSC) of diclofenac sodium, metronidazole hydrochloride and microspheres were performed using Perkin-Elmer DSC-7 model. The instrument was calibrated with indium. All the samples $(\approx 5 \mathrm{mg})$ were heated in sealed aluminum pans with perforated lids using dry nitrogen as the effluent gas. The analysis was performed in the temperature range of $50-400{ }^{\circ} \mathrm{C}$ and at a rate of $20^{\circ} \mathrm{C} \mathrm{min}{ }^{-1}$.

\section{Evaluation of in vitro release by static method}

Microspheres, equivalent to $10 \mathrm{mg}$ of diclofenac sodium, were accurately weighed and transferred to $250 \mathrm{ml}$ conical flask containing $100 \mathrm{ml}$ phosphate buffer (pH 7.2). The flask was kept in an incubator at $37^{\circ} \mathrm{C}, 1$ $\mathrm{ml}$ samples withdrawn at regular intervals and, after suitable dilution, the amount of drug released was determined using a spectrophotometer at $276 \mathrm{~nm}$. Following each sample withdrawal, $1 \mathrm{ml}$ of phosphate buffer was added to the release medium to replenish it. Five minutes before each sampling, the flasks were gently shaken by manually whirling it clockwise (15 revolutions) to minimize any concentration gradient within the release medium. The microspheres were allowed to settle down and clear supernatant medium withdrawn for drug analysis. The sample was filtered and the microspheres collected were transferred to the dissolution flask. Similarly, the release of metronidazole hydrochloride from gelatin microspheres was determined spectrophotometrically at $320 \mathrm{~nm}$ (Shimadzu 1601).

\section{Evaluation of in vitro release by dynamic method}

Evaluation in vitro release studies of diclofenac sodium or metronidazole hydrochloride from the carbomer gel was carried out at $37^{\circ} \mathrm{C}$ using phosphate buffer $(\mathrm{pH} 7.2)$ as the release medium. A glass tube of $10 \mathrm{~mm}$ diameter and $100 \mathrm{~mm}$ height was taken. One end of the tube was closed using an egg membrane with the help of adhesive tape while the other end was kept open and used as drug reservoir compartment. Gel (1 g) containing diclofenac sodium was accurately weighed and transferred to the glass tube in a vertical position through the open end. The gel was gently pushed down to the surface of the egg membrane with the help of a stainless steel spatula to ensure that all the gel was in contact with the membrane. Phosphate buffer $(2 \mathrm{ml}, \mathrm{pH} 7.2)$ was added to the reservoir compartment to wet the gel. The glass tube was placed in a beaker containing $100 \mathrm{ml}$ of phosphate buffer (pH 7.2) such that that the egg membrane is just immersed in the phosphate buffer which acted as the receiving compartment. The receiving compartment was magnetically stirred (100 rpm, Remi, India) at $37^{\circ} \mathrm{C}$. Samples $(1 \mathrm{ml})$ were withdrawn from the receiving compartment at regular intervals and the amount of diclofenac sodium released from the gel was determined using a spectrophotometer at $276 \mathrm{~nm}$ (Shimadzu 1601). After each withdrawal of sample, an 
equal quantity of phosphate buffer was added to the receiving compartment to replenish it. Similarly metronidazole hydrochloride release from the gel was determined spectrophotometrically at $320 \mathrm{~nm}$.

\section{Release kinetics}

Data obtained from in vitro release studies were fitted to various kinetic equations [11] to determine the mechanism of drug release from the microspheres. The kinetic models used were zero order equation, first order equation and Higuchi release using the following plots: $Q_{t}$ vs $t, \log \left(Q_{0}-Q_{t}\right)$ vs $t$ and $Q_{t}$ vs square root of $t$, respectively; where $Q_{t}$ is the amount of drug released at time $t$ and $Q_{0}$ is the initial amount of drug present in the microspheres. $T$ further, to ascertain the mechanism of drug release, the first $60 \%$ of drug release was fitted to Korsmeyer-Peppas model (Eq 3).

$$
M_{t} / M_{\alpha}=k t^{n}
$$

where $M_{t} / M_{\alpha}$ is the fraction of drug released at time, $t, k$ is the rate constant and $n$ is the release exponent. The $n$ value is used to characterize different release mechanisms.

\section{Statistical analysis}

Drug loading, entrapment and encapsulation efficiency data were analyzed by one sample T-test while drug release data were analyzed by two-way ANOVA. PASW 18 statistics software (IBM, USA) was used for statistical analysis and confidence level was set at $p<$ 0.05 .

\section{RESULTS}

\section{Drug loading, entrapment and encapsu- lation efficiency}

The loading, entrapment and encapsulation efficiencies of the microspheres were 23.4, 93.6 and $74.9 \%$, respectively, for diclofenac and $21.5,86.0$ and $73.1 \%$, respectively, for metronidazole. There was no significant difference $(p>0.05)$ between two drugs with regard to these parameters.

\section{Particle size of microspheres}

The size distribution profiles of the microspheres are shown in Figure 1. The particle size of unloaded microspheres ranged from 1 - $50 \mu \mathrm{m}$ compared with 10$110 \mu \mathrm{m}$ for the drug-loaded microspheres. Mean diameter of unloaded, diclofenac sodium-loaded and metronidazole hydrochloride-loaded microspheres was $33.5,67.8$ and $51.4 \mu \mathrm{m}$, respectively.

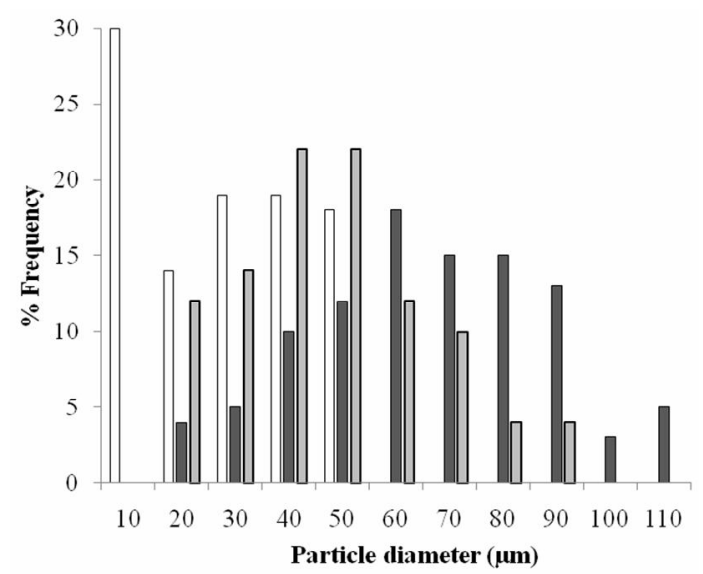

Figure 1: Particle size distributions of unloaded $(\square)$, diclofenac sodium-loaded ( $\square$ ) and metronidazole hydrochloride-loaded $(\square)$ gelatin microspheres.

\section{Scanning electron microscopy}

The microspheres exhibited good spherical geometry as evident by the SEM photomicrographs in Figure 2. However, diclofenac sodium microspheres were larger size and showed less aggregation than metronidazole microspheres.

\section{Differential scanning calorimetry}

The thermogram of unloaded microspheres showed no peaks while the thermogram of diclofenac sodium showed a sharp peak at its melting point $\left(276{ }^{\circ} \mathrm{C}\right)$. The physical mixture of unloaded microspheres and diclofenac 
sodium showed a less prominent peak at 276 ${ }^{\circ} \mathrm{C}$ but the peak was absent in diclofenac sodium-loaded microspheres (Figure 3 ).

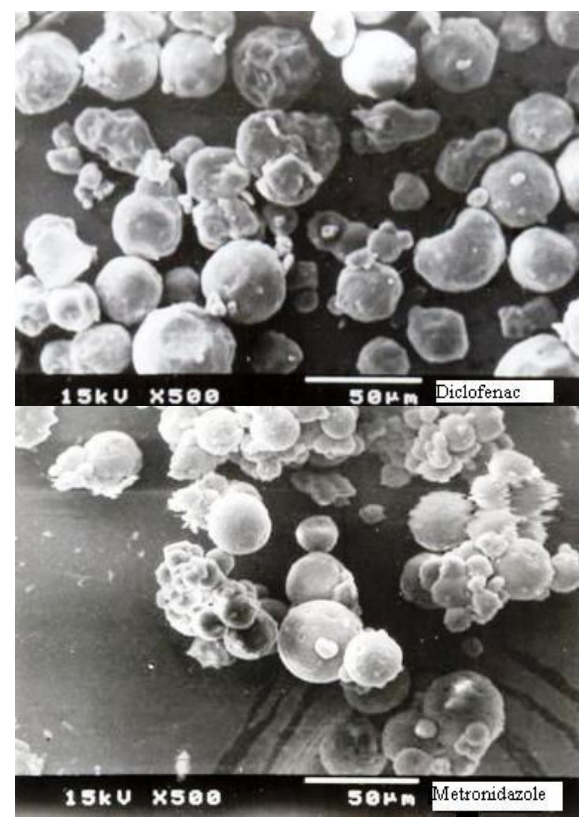

Figure 2: SEM photographs of gelatin microspheres loaded with diclofenac sodium (left) and metronidazole hydrochloride (right).

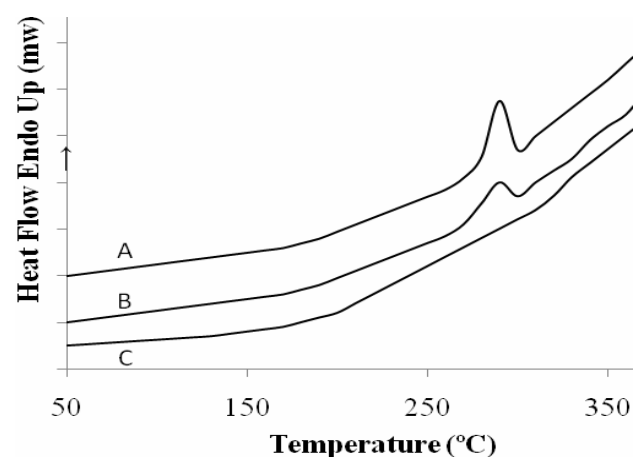

Figure 3: DSC thermograms of $(A)$ diclofenac sodium, (B) physical mixture (1:1) of diclofenac sodium and unloaded gelatin microspheres and (C) gelatin microspheres loaded with diclofenac sodium

Similarly, the thermogram of metronidazole hydrochloride showed a sharp peak at its melting point of $160{ }^{\circ} \mathrm{C}$ while the physical mixture of unloaded microspheres and metronidazole hydrochloride showed a less prominent at $160{ }^{\circ} \mathrm{C}$ and the peak was absent in metronidazole-loaded microspheres.

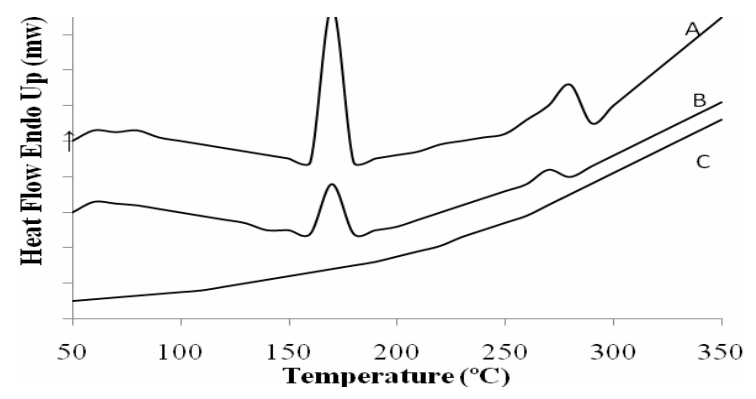

Figure 4: DSC thermograms of $(A)$ metronidazole hydrochloride, (B) physical mixture (1:1) of metronidazole hydrochloride and unloaded gelatin microspheres and $(\mathrm{C})$ gelatin microspheres loaded with metronidazole hydrochloride

\section{In vitro drug release}

The cumulative release of diclofenac sodium and metronidazole from microspheres and gels are shown in Figure 5. Drug from the microspheres was released in a controlled manner for $>4 \mathrm{~h}$. The release pattern was biphasic with an initial 'burst' release of $34 \%$ of the loaded drug was in the first $10 \mathrm{~min}$. Thereafter, release was slow but steady, and by the end of the $4^{\text {th }}$ hour, $85 \%$ of the loaded drug was released.

Diclofenac release from the carbomer gel formulation is also shown in Figure 5. The release pattern was biphasic with an initial 'burst' release that was enhanced by the loading dose of diclofenac sodium. By the end of the first $10 \mathrm{~min}, 45$ to $48 \%$ of the drug was released; subsequently, release was steady and by the end of the $4^{\text {th }} \mathrm{h}, 90 \%$ of the drug was released. The data for the microsphere and gel containing metronidazole are similar with slow drug release that reached a plateau in $3-4 \mathrm{~h}$. There was significant difference $(p<0.05)$ between the release rates of diclofenac sodium and 

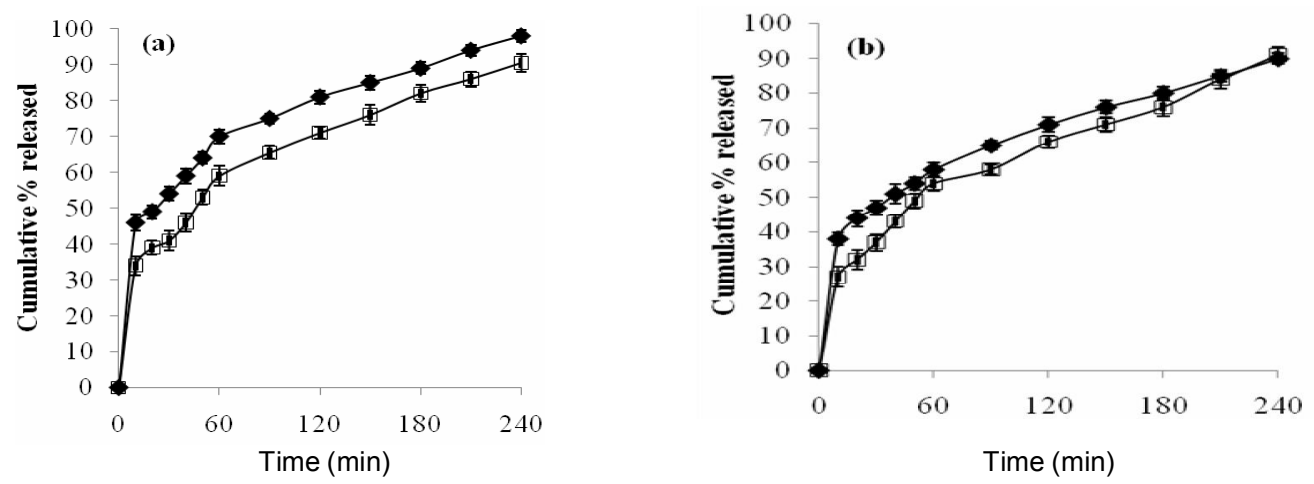

Figure 5: In vitro release of (a) diclofenac sodium and (b) metronidazole from gelatin microspheres ( $\square$ ) and microspheres-loaded gel $(\diamond) ;(n=3)$.

metronidazole from microspheres with the former greater. A similar difference between the two drugs was observed with regard to the formulated gels.

\section{Release kinetics}

Drug release from the microspheres followed more closely the Higuchi model with a regression coefficient $\left(r^{2}\right)$ of 0.9793 compared with first order $\left(\left(r^{2}=0.9522\right)\right.$ and zero order models $\left(r^{2}=0.8289\right)$. Subjection of the release data to Korsmeyer- Peppas analysis yielded $n$ value of 0.37 for diclofenac sodium and 0.38 for metronidazole hydrochloride.

\section{DISCUSSION}

Sesame oil containing $0.1 \% \mathrm{v} / \mathrm{v}$ Span 80 as dispersing medium produced drug-loaded gelatin microspheres with good loading and spherical geometry. The addition of surfactant contributed to the good spherical geometry and reduced aggregation of the microspheres. Diclofenac sodium was better encapsulated in gelatin microspheres than the metronidazole and this could be attributed to the high protein binding nature of diclofenac sodium [6].

The microspheres were incorporated in a conventional carbomer gel formulation [7]. Carbopol 934 is a commercial grade of carbomer. It is gelled by neutralization with triethanolamine. Methyl hydroxylbenzoate and propyl hydroxylbenzoate are used as antimicrobial preservative; while sodium metabisulphite is used as an anti-oxidant. The formulated carbomer gel was very clear, transparent and elegant. Incorporation of gelatin microspheres resulted in finely dispersed particles in the gel system. A loading dose of the free drug was incorporated in the carbomer gel to produce immediate effect while drug-loaded microspheres are added to provide sustaining dose.

Volume-surface mean diameter microspheres is important in drug delivery because it is inversely related to the specific surface area [13] and hence the release rate. Larger the diameter, slower the drug release and vice versa.

Drug-loaded microspheres were larger in size than the unloaded ones. Diclofenac sodium loaded microspheres were larger than metronidazole-loaded microspheres. Crosslinking of gelatin with glutaraldehyde mormally would shrink the polymeric network [14], resulting in the reduction of particle size. The greater the degree of cross-linking the smaller the diameter microspheres. Perhaps, there was less cross-linking with diclofenac sodium than with metronidazole during microsphere formation. Particle diameter is 
an important factor influencing drug release and retention in target tissue.

The size of periodontal pockets varies between 1 to $4 \mathrm{~mm}$ depending on the disease condition [4]. Particle sizes between 20 to $120 \mu \mathrm{m}$ have been suggested to be suitable for periodontal applications [15]. Thus the sizes of the microspheres were within the desired range.

Previous FT-IR studies conducted in our laboratory indicate that there was no interaction between gelatin and metronidazole [12] or diclofenac sodium $[10,14]$. The smaller peaks in the DSC of the physical mixture of unloaded microspheres and metronidazole/diclofenac sodium compare with those of the individual drugs is due to low quantities of the drugs in the physical mixture. The completely absence of crystalline drug peaks in the microsphere formulation, like in the FT-IR spectra, indicates the transformation of the drugs from crystalline to amorphous state.

It is difficult to mimic the physiological environment in periodontal pockets. The drug release study was performed in both static and dynamic environments. Drug release from gelatin microspheres based on the static model was designed to mimic the static state inside the carbomer gel. The egg membrane pouch was used as a model for periodontal pocket in order to determine the diffusion of drug across biological membrane. Both the microspheres and gel formulations sustained the drug release more than $4 \mathrm{~h}$, indicating the capacity of the formulated gel and microspheres to release the drugs for a prolonged period. The burst drug release produced by both the microspheres and the gel formulation would be beneficial in getting achieving rapid onset of action. Burst release was approx. $10 \%$ higher for gel than for microspheres. Thus, the burst and sustained release pattern of the gel formulation will be useful in treating inflammation and infection locally in periodontal cavities. Formulation of diclofenac sodium showed faster release than the metronidazole. Diclofenac sodium formulation showed faster release than metronidazole formulation due probably to the higher solubility and lower degree of cross-linking of the former at $\mathrm{pH} 7.2$.

Higher regression coefficient was obtained with Higuchi than with first order and zero order. These findings indicate that drug release from the microspheres was diffusioncontrolled. With time, drug release rate fell as the drug molecule had to diffuse through the longer pathway in cross-linked gelatin matrix. The $n$ value obtained from the release data of microspheres suggests that drug mechanism was pseudo-Fickian diffusion.

\section{CONCLUSION}

The formulated microspheres showed good drug-loading. The gel formulation, which consisted of drug-loaded gelatin microspheres, showed sustained release of diclofenac sodium and metronidazole hydrochloride for $\geq 4 \mathrm{~h}$, thus indicating their suitability for the sustained delivery of the drugs for the treatment of inflammation and infections in periodontal pockets. However, further studies, including clinical tests are required to confirm the gel's therapeutic efficacy.

\section{REFERENCES}

1. Listgarten MA. Pathogenesis of periodontitis. J Clin Periodontol 1986; 13(5): 418-425.

2. Listgarten MA. Nature of periodontal diseases: Pathogenic mechanisms. J Periodontal Res 1987; 22(3): 172-178.

3. Van der Ouderaa FJG. Anti-plaque agents. Rationale and prospects for prevention of gingivitis and periodontal disease. $J$ Clin Periodontol 1991; 18(6): 447-454.

4. Renvert S, Lessem J, Dahlen G, Lindahi C Svensson M. Topical minocycline microspheres versus topical chlorhexidine gel as an adjunct to mechanical debridement of incipient peri-implant infections: a randomized clinical trial. J Clin Periodontol 2006; 33(5): 362-369.

5. Jain N, Jain GK, Javed S, Iqbal Z, Talegaonkar S, Ahmed FJ, Khar RK. Recent approaches for the treatment of periodontitis. Drug Discov Today 2008; 13(21-22): 932-943. 
6. Dollery C (Ed) Therapeutic Drugs, Volume 1, edn 2, London: Churchill Livingstone; 1999. pp. D88D91.

7. Carter SJ, Cooper and Gunn's dispensing for pharmaceutical students. edn 12. New Delhi: CBS Publisher; 1987. pp 216-217.

8. Fu J, Sun $X$, and Z. ZR, Study on bioadhesive property of carbomer 934 by a gamma camera in vivo. World J Gasteroenterology 2002; 8(1): 176-179.

9. Varshosaz J, Tavakoli N, Saidian S. Development and physical characterization of a periodontal bioadhesive gel of metronidazole. Drug Deliv 2002; 9: 127-133.

10. Callens C, Pringels E, Remon JP, Influence of multiple nasal administrations of bioadhesive powders on the insulin bioavailability. Int $J$ Pharm 2003; 250(2): 415-422.

11. Saravanan M, Bhaskar K, Maharajan G, Sadasivan Pillai K. Ultrasonically controlled release and targeted delivery of diclofenac sodium via gelatin magnetic microspheres. Int $\mathrm{J}$ Pharm 2004; 283(1-2): 71-82.
12. Saravanan M, Panduranga Rao K. Pectin-gelatin and alginate-gelatin complex coacervation for controlled drug delivery: Influence of anionic polysaccharides and drugs being encapsulated on physicochemical properties of microcapsules. Carbohyd Polym 2010; 80(3): 808-816.

13. Sinko PJ (Ed) Martin's Physical Pharmacy and Pharmaceutical Sciences, edn 5. Baltimore: Lippincott Williams and Wilkins; 2006. pp 518519.

14. Saravanan M, Bhaskar K, Maharajan G, Sadasivan Pillai K. Development of gelatin microspheres loaded with diclofenac sodium for intraarticular administration. J Drug Target 2011; 19(2): 96-103.

15. Cortesi R, Menegatti E, Esposito, Nastruzzi C. Microparticles as drug delivery systems for local delivery to the oral cavity. In: Rathbone MJ, Hadgraft J, Roberts MS, Eds. Modified Release Drug Delivery Technology, edn 1, New York: Marcel Dekker; 2003; pp 453-454. 